\title{
Integration in response timing: The functional time requirement
}

\author{
MICHAEL D. ZEILER \\ Emory University, Atlanta, Georgia
}

\begin{abstract}
Two procedures involving differential reinforcement of the duration of fixed-ratio sequences required pigeons to choose between simultaneously available duration requirements. One variation demanded only that the ratio durations exceed a lower limit; the second entailed an upper limit as well. In the no-upper-bound conditions, ratio duration was influenced by the other choice option. The larger the difference between the two duration requirements, the more ratio duration in one component shifted in the direction of the other requirement. The upper-bound procedure weakened this interaction. The temporal differentiation equation-an equation that has successfully described the effects of single time requirements on a variety of species and response units-fit the present results when the functional time requirement was viewed as the weighted average of the component requirements. The weighting parameter determining the functional requirement was influenced by the presence or absence of the upper bound. As has proven generally the case in temporal differentiation experiments, behavior did not conform to Weber's law. Instead, sensitivity decreased as time taken to complete the ratio increased.
\end{abstract}

Temporal differentiation procedures require that animals time their behavior in order to obtain reinforcement. These procedures involve the psychophysical method of production, in which the emitted response must conform to a duration requirement and feedback consists of the occurrence or nonoccurrence of the reinforcer. In situations demanding that emitted duration exceed some requirement, the data are well described by the power function $T=k t^{n}$, where $T$ is emitted duration, $t$ is the requirement, and $k$ and $n$ are fitting parameters, whether the behavior involved was barpress duration in rats, response latency in pigeons, or the duration of extended response sequences in pigeons (DeCasper \& Zeiler, 1977; Platt, 1979). Although the values of $k$ and $n$ varied across experiments, these variations turned out to be nonarbitrary. DeCasper and Zeiler (1977) found that the two parameters were predicted by the duration emitted in the absence of time requirements (the base duration), and they proposed the equation $\mathrm{T}=1.6 \mathrm{~d}^{.18} \mathrm{t}^{.82-.05} \mathrm{in} \mathrm{d}$ to describe the results. (This equation differs from that given by DeCasper and Zeiler because of the substitution of natural for common logs.) The equation-referred to as the temporal differentiation equation-has no fitting parameters: $T$ is emitted duration, $t$ is the minimum duration requirement,

This research was supported by Orant BNS 80-23248 from the National Science Foundation. I am indebted to Gregory $\mathbf{K}$. Scott for our hours of fruitful conversation about timing and this paper. Reprints may be obtained from Michael D. Zeiler, Department of Psychology, Emory University, Atlanta, Georgia 30322. $\mathrm{d}$ is the duration occurring without a time requirement, and the other terms are constants. The equation successfully described the majority of data on response timing. The rational basis of the equation is that the power function parameters are determined by base duration (d); the particular numerical constants are estimations derived from the previous data.

Despite the success of the temporal differentiation equation, other results indicate that it cannot be generally correct. All of the confirming experiments used a procedure involving only a minimum duration requirement. However, the addition of an upper bound alters the nature of timing (Kuch, 1974), thereby showing that consideration of only the lower bound must be inadequate. Apparently, the lower bound does not fully describe the functional (effective) time requirement. If, in fact, the functional requirement is the reinforced duration rather than the lower bound (which operates to set the lower limit for reinforcement), the various data can be reconciled. The relation between reinforced and all emitted durations can also be described by a power function (Gibbon, 1977), and a form of the parameter-free temporal differentiation equation also applies. The reinforced duration version of the equation is approximated by $T=1.4 \mathrm{~d}^{.15}$ $R^{.44-05 i n d}$, where $R$ is reinforced duration and the other terms are as described above. The constants of the two forms of the temporal differentiation equation are so similar because, for the studies involving no upper bounds, reinforced duration typically was a simple transform of the time requirement. Upper bounds change the reinforced durations, thereby 
allowing the reinforced duration version of the equation to handle the results while the lower bound version could not. The reinforced duration version has the additional advantage of conforming to the intuitively reasonable idea that these durations are the only source of feedback to the animal about the nature of the prevailing time requirement. A dynamic relation between experienced reinforced duration and ongoing behavior would be compatible with much of what is known about operant behavior in general. These considerations lead to the hypothesis that the functional time requirement is the experienced reinforced duration.

A next step in developing a comprehensive account of response timing is to add to the complexity of the situation. The present experiment investigated whether the formulations adequate to the simple cases would apply when an animal had to deal with two duration requirements. Requirements involving sequence durations (the time taken to begin and complete a fixed ratio) exceeding a criterion were imposed simultaneously, and the pigeons had to choose between them on each trial. If pigeons showed orderly behavior under such conditions, three effects could occur. One would be independence of the two requirements. The other two possibilities would be interactions: each requirement could affect the other. The interaction could take the form of contrast, in which the durations controlled by each requirement became more widely separated than would be expected, or of induction, in which the difference between the two durations was reduced. The occurrence of interactions potentially could separate the predictions of the two forms of the temporal differentiation equation. The reinforced version would describe the data more satisfactorily if both the reinforced and remaining durations occurring in a component were changed by the presence of an accompanying additional time requirement, because the lower bound version has no way of dealing with such interactions.

Interactions would be important for another reason as well. Their occurrence would mean that the effects of a given time requirement were not constant but were influenced by other features of the experimental situation. Such contextual influences on timing would fit with Helson's (1964) assertion that the effective stimulus always represented the combination of current stimulation, previous stimulation, and background as well as with Anderson's (1981) insistence that the subject's integration of nominally separate experiences typically determined the effects of any one stimulus. Interactions in the present study would mean that behavior under one requirement was determined not only by that requirement, but by the other as well. Solving the temporal differentiation equations for $t$ and $R$ would then indicate the nature of the functional controlling value. The relation of the functional to the two nominal values potentially could show how the separate values were integrated.

The procedure always involved a minimumduration requirement, and also involved the presence or absence of an upper bound. One reason for this manipulation was that previous experiments on fixed-ratio duration involved only lower bounds, but data on other response units had shown that upper limits altered the nature of timing (Kuch, 1974). The second reason was to determine if this feature affected any interactions that might occur between simultaneous time requirements.

\section{METHOD}

\section{Subjects}

Four adult White Carneaux pigeons were maintained at $80 \%$ of their free-feeding weights. Birds 16 and 110 had had prior experience with a variety of reinforcement schedules and discriminative stimuli, and Birds 143 and 159 were experimentally naive.

\section{Apparatus}

The experimental chamber was a standard two-key pigeon unit. The keys (R. Gerbrands Co.) operated with a minimum force of $.18 \mathrm{~N}$. The left key could be transilluminated by two blue, white, or red 7-W lights, and the right key by two green, white, or red 7-W lights. The reinforcer was 3-sec access to Purina Pigeon Checkers, the birds' standard diet. The hopper aperture was illuminated by a 1 -W white light during food deliveries. All experimental events were programmed and recorded by an on-line computer. Continuous white noise helped mask extraneous sounds.

\section{Procedure}

Birds 16 and 110 required no shaping, but Birds 143 and 159 were shaped by successive approximations to peck both keys when blue (left key) and green (right key). After the requirement for food delivery via each key was raised to 30 responses, the experiment proper began.

Each trial began with the left key blue and the right key green. The first peck to either key darkened the other and made responses to it ineffective. Twenty-nine more responses to the lit key completed the ratio. Choice, therefore, could occur only for the first response; the bird had only one option after that response.

Reinforcer assignment. Food was available for completing the FR 30 on only one of the keys on any trial. At the beginning of each session and after each food delivery, the next reinforcer was assigned to each key with $p=.5$. The assignment remained until food delivery. This arrangement guaranteed that no matter how often the pigeon chose each key, the number of food deliveries via each would be approximately equal. A further assurance was provided by having the next reinforcer assigned to one key whenever the second key had produced three more reinforcers than the first in the session. The intent was to prevent the development of exclusive preference for one alternative. The choice data shown in Tables 1 and 2 (ratios completed on each key, reinforcers derived from each key) show that the procedure was effective in this regard.

Duration requirements: No upper bound. Table 1 shows the first sequence of conditions. In the first condition (lower bound = $0 \mathrm{sec}$ ), if the pigeon chose the key to which food delivery was assigned, completion of the FR 30 turned off the key light (blue 
Table 1

No Upper Bound : Sequence of Conditions

\begin{tabular}{|c|c|c|c|c|c|c|}
\hline \multirow{2}{*}{$\begin{array}{l}\text { Condi- } \\
\text { tion }\end{array}$} & \multicolumn{2}{|c|}{$\begin{array}{l}\text { Lower Bound } \\
\text { (in Seconds) }\end{array}$} & \multicolumn{2}{|c|}{ Ratios } & \multicolumn{2}{|c|}{ Reinforcers } \\
\hline & Left & Right & Left & Right & Left & Right \\
\hline \multicolumn{7}{|c|}{ Bird 16} \\
\hline 1 & 0 & 0 & 137 & 103 & 73 & 77 \\
\hline 2 & 12 & 12 & 263 & 237 & 71 & 72 \\
\hline 3 & 15 & 15 & 271 & 229 & 65 & 60 \\
\hline 4 & 21 & 15 & 222 & 278 & 61 & 57 \\
\hline 5 & 30 & 45 & 238 & 262 & 38 & 33 \\
\hline 6 & 15 & 60 & 231 & 269 & 23 & 21 \\
\hline 7 & 18 & 15 & 192 & 308 & 49 & 57 \\
\hline 8 & 45 & 60 & 233 & 267 & 25 & 26 \\
\hline 9 & 21 & 63 & 288 & 212 & 27 & 22 \\
\hline 10 & 18 & 18 & 219 & 281 & 58 & 59 \\
\hline 11 & 15 & 30 & 273 & 227 & 29 & 29 \\
\hline \multicolumn{7}{|c|}{ Bird 110} \\
\hline 1 & 0 & 0 & 131 & 113 & 71 & 79 \\
\hline 2 & 12 & 12 & 228 & 185 & 79 & 71 \\
\hline 3 & 15 & 15 & 189 & 256 & 71 & 79 \\
\hline 4 & 15 & 60 & 172 & 328 & 27 & 21 \\
\hline 5 & 18 & 18 & 187 & 194 & 77 & 73 \\
\hline 6 & 15 & 30 & 188 & 226 & 77 & 73 \\
\hline 7 & 30 & 45 & 228 & 272 & 35 & 33 \\
\hline 8 & 18 & 15 & 225 & 178 & 78 & 72 \\
\hline 9 & 21 & 15 & 122 & 193 & 71 & 79 \\
\hline 10 & 63 & 21 & 318 & 182 & 21 & 21 \\
\hline 11 & 15 & 30 & 196 & 262 & 73 & 77 \\
\hline \multicolumn{7}{|c|}{ Bird 143} \\
\hline 1 & 0 & 0 & 117 & 122 & 75 & 75 \\
\hline 2 & 12 & 12 & 146 & 168 & 68 & 82 \\
\hline 3 & 15 & 15 & 141 & 219 & 71 & 79 \\
\hline 4 & 45 & 30 & 302 & 198 & 12 & 14 \\
\hline 5 & 15 & 18 & 194 & 306 & 67 & 66 \\
\hline 6 & 15 & 21 & 225 & 275 & 57 & 61 \\
\hline 7 & 60 & 15 & 365 & 135 & 15 & 18 \\
\hline 8 & 18 & 18 & 265 & 235 & 49 & 45 \\
\hline 9 & 30 & 15 & 283 & 217 & 29 & 34 \\
\hline 10 & 45 & 15 & 421 & 79 & 20 & 21 \\
\hline 11 & 63 & 21 & 238 & 262 & 19 & 20 \\
\hline \multicolumn{7}{|c|}{ Bird 159} \\
\hline 1 & 0 & 0 & 168 & 86 & 81 & 69 \\
\hline 2 & 12 & 12 & 287 & 213 & 51 & 57 \\
\hline 3 & 15 & 15 & 298 & 202 & 32 & 27 \\
\hline 4 & 15 & 18 & 284 & 216 & 34 & 32 \\
\hline 5 & 15 & 21 & 238 & 262 & 23 & 27 \\
\hline 6 & 60 & 15 & 370 & 130 & 8 & 10 \\
\hline 7 & 30 & 15 & 246 & 254 & 16 & 21 \\
\hline 8 & 45 & 15 & 327 & 173 & 9 & 12 \\
\hline 9 & 18 & 18 & 237 & 263 & 33 & 32 \\
\hline 10 & 45 & 30 & 200 & 300 & 7 & 6 \\
\hline 11 & 21 & 63 & 283 & 217 & 8 & 8 \\
\hline
\end{tabular}

or green), turned on the white light on that key for $.5 \mathrm{sec}$, and then operated the food hopper for $3 \mathrm{sec}$. If the other key was chosen, the FR 30 was followed by $.5 \mathrm{sec}$ of white.

Subsequent conditions involved duration requirements. The time taken from trial onset to completion of the FR 30 had to meet or exceed that specified to be eligible for food delivery. The time to the first response (the response that committed the pigeon to a given key on that trial) counted as time allocated to the chosen key. If the duration of the ratio met the requirement, the sequence of events was as described for the first condition. If the emitted duration did not meet the time require- ment, completion of the FR 30 was followed by $3 \mathrm{sec}$ with the ke:- red. Table 1 shows that as one of the time requirements increased, the number of reinforcers per session decreased.

Duration requirements: Upper bound. Table 2 shows the next sequence of conditions. The distinctive aspect was the addition of upper limits to each duration requirement. The ratio duration had to equal or exceed the lower bound and be shorter than the upper bound to be eligible for food delivery. The width of the reinforceable bin was one-third of the lower bound.

The sequence of events was exactly as before. The key became white for $.5 \mathrm{sec}$ following ratios meeting the duration requirement, and then food delivery occurred if it had been assigned to that key for the trial. It became red for $3 \mathrm{sec}$ if the duration was incorrect (now either too long or too short). Once again, as the lower bound increased, the number of reinforcers per session decreased.

Other features. Each session (sessions were conducted 5 days per week) lasted for 30 food deliveries or for 100 ratios, whichever occurred first. Each condition was maintained for 30 sessions, by which time mean ratio duration showed no monotonic direction of change over the last five sessions, and the five individual means for each key were within $5 \%$ of the overall means. One exception was that Bird 143 failed to stabilize in 8 of the 10 conditions involving upper bounds, and all of the upperbound data were excluded from subsequent analyses, because the remainder were too scanty to allow quantitative treatment. The only other exception was that Bird 110 failed to stabilize in two upper-bound conditions. Because the other eight conditions provided a good range of time requirements, only the two unstable conditions were excluded from subsequent treatment.

\section{RESULTS}

Data analyses were based on the last five sessions of a condition. The duration emitted on a given key appeared to be independent of what had occurred previously. The durations were the same whether a food delivery had occurred on either that key or the other, or whether the preceding duration had been followed by the signal indicating either correctness or incorrectness. Sequential dependencies also were not apparent in choices of each key. The mean of all ratio times, their standard deviations, the mean reinforced durations, and their standard deviations appear in Tables 3 and 4.

\section{Mean Ratio Durations: Quantitative Analysis}

The data were approached in several ways. A standard analysis of response timing is to determine the fitting parameters ( $k$ and $n$ ) of the power function $\left(T=\mathbf{k t}^{n}\right)$ relating emitted duration $(T)$ to the duration requirement $(t)$. The second approach involved the lower bound version of the temporal differentiation equation. The third strategy was to consider the two dependent variables of mean response duration and mean reinforced response duration.

Figures 1 and 2 show the mean ratio times plotted against the lower bound of the time requirement. Table 5 gives the parameters and goodness of fit of the best-fitting power functions based on the percent variance accounted for by the residual mean 
Table 2

Upper Bound: Sequence of Conditions

\begin{tabular}{|c|c|c|c|c|c|c|}
\hline \multirow[b]{2}{*}{ Condition } & \multicolumn{2}{|c|}{$\begin{array}{l}\text { Lower Bound-Upper Bound } \\
\text { (in Seconds) }\end{array}$} & \multicolumn{2}{|c|}{ Ratios } & \multicolumn{2}{|c|}{ Reinforcers } \\
\hline & Left & Right & Left & Right & Left & Right \\
\hline \multicolumn{7}{|c|}{ Bird 16} \\
\hline 1 & $0-\infty$ & $0-\infty$ & 121 & 130 & 74 & 76 \\
\hline 2 & $15-20$ & $15-20$ & 224 & 276 & 46 & 44 \\
\hline 3 & $21-28$ & $63-84$ & 265 & 235 & 18 & 18 \\
\hline 4 & $63-84$ & $30-40$ & 264 & 236 & 8 & 7 \\
\hline 5 & $15-20$ & $30-40$ & 255 & 245 & 34 & 37 \\
\hline 6 & $45-60$ & $21-28$ & 285 & 215 & 15 & 20 \\
\hline 7 & $15-20$ & $15-20$ & 261 & 239 & 55 & 53 \\
\hline 8 & $15-20$ & 4560 & 278 & 222 & 21 & 19 \\
\hline 9 & $45-60$ & $15-20$ & 235 & 265 & 24 & 22 \\
\hline 10 & $15-20$ & $63-84$ & 259 & 241 & 17 & 17 \\
\hline 11 & $30-40$ & 4560 & 230 & 270 & 7 & 10 \\
\hline \multicolumn{7}{|c|}{ Bird 110} \\
\hline 1 & $0-\infty$ & $0-\infty$ & 130 & 115 & 76 & 74 \\
\hline 2 & $15-20$ & $30-40$ & 234 & 266 & 54 & 56 \\
\hline 3 & $15-20$ & 4560 & 237 & 263 & 13 & 10 \\
\hline 4 & $15-20$ & $63-84$ & 245 & 255 & 8 & 12 \\
\hline 5 & $45-60$ & $15-20$ & 248 & 252 & 24 & 24 \\
\hline 6 & $21-28$ & $45-60$ & 254 & 246 & 13 & 9 \\
\hline 7 & $21-28$ & 15.20 & 278 & 222 & 38 & 43 \\
\hline 8 & $15-20$ & $15 \cdot 20$ & 254 & 246 & 43 & 41 \\
\hline 9 & $63-84$ & $21.28^{*}$ & 202 & 298 & 18 & 17 \\
\hline 10 & $21-28$ & $30-40$ & 215 & 285 & $\begin{array}{l}16 \\
16\end{array}$ & 14 \\
\hline 11 & $15-20$ & $45-60^{*}$ & 244 & 256 & 14 & 13 \\
\hline \multicolumn{7}{|c|}{ Bird 143} \\
\hline 1 & $0-\infty$ & $0-\infty$ & 183 & 119 & 76 & 74 \\
\hline 2 & $30-40$ & $15-20^{*}$ & 354 & 146 & 12 & 13 \\
\hline 3 & $45-60$ & $15-20^{*}$ & 403 & 97 & 6 & 5 \\
\hline 4 & $63-84$ & $15-20 *$ & 288 & 212 & 9 & 10 \\
\hline 5 & $45-60$ & $15-20 *$ & 396 & 104 & 8 & 10 \\
\hline 6 & $15-20$ & $15-20$ & 288 & 212 & 29 & 31 \\
\hline 7 & $15-20$ & $3040^{*}$ & 276 & 224 & 13 & 13 \\
\hline 8 & $30-40$ & $15-20 *$ & 367 & 133 & 17 & 14 \\
\hline 9 & $15-20$ & $4560 *$ & 204 & 296 & 14 & 10 \\
\hline 10 & 63-84 & $21-28 *$ & 309 & 191 & 7 & 9 \\
\hline 11 & $21-28$ & $30-40$ & 327 & 173 & 17 & 12 \\
\hline \multicolumn{7}{|c|}{ Bird 159} \\
\hline 1 & $0-\infty$ & $0-\infty$ & 120 & 108 & 75 & 75 \\
\hline 2 & $15-20$ & $15-20$ & 277 & 223 & 14 & 13 \\
\hline 3 & $21-28$ & $45-60$ & 334 & 166 & 10 & 8 \\
\hline 4 & 63-84 & $15-20$ & 265 & 235 & 10 & 8 \\
\hline 5 & $15-20$ & $21-28$ & 302 & 198 & 30 & 24 \\
\hline 6 & $21-28$ & 63.84 & 389 & 111 & 9 & 10 \\
\hline 7 & $15-20$ & 4560 & 301 & 199 & 11 & 9 \\
\hline 8 & $30-40$ & $15-20$ & 304 & 196 & 7 & 10 \\
\hline 9 & $45-60$ & $15-20$ & 312 & 188 & 9 & 13 \\
\hline 10 & $21-28$ & $30-40$ & 307 & 193 & 14 & 13 \\
\hline 11 & $15-20$ & $30-40$ & 317 & 183 & 12 & 10 \\
\hline
\end{tabular}

*Unstable behavior in these conditions.

square (McDowell, 1981). Fits were better for the upper-bound than for the no-upper-bound conditions. Table 5 also shows that the temporal differentiation equations accounted for about the same amount of variance as did the power functions in the upper-bound conditions, but did less well in the no-upper-bound conditions. With respect to the two forms of the temporal differentiation equa- tion, the reinforced duration version consistently provided the better fit to the no-upper-bound conditions.

Figures 3 and 4 show mean ratio time plotted against mean reinforced ratio time, and Table 5 gives the parameters of the best-fitting power functions. The total ratio times fell progressively shorter of matching the reinforced times as the latter in- 
Table 3

No Upper Bound: Results

\begin{tabular}{|c|c|c|c|c|c|c|c|c|c|}
\hline & & \multicolumn{4}{|c|}{ Ratio Time } & \multicolumn{4}{|c|}{ Reinforced Ratio Time } \\
\hline \multicolumn{2}{|c|}{ Lower Bound } & \multicolumn{2}{|c|}{ Left } & \multicolumn{2}{|c|}{ Right } & \multicolumn{2}{|c|}{ Left } & \multicolumn{2}{|c|}{ Right } \\
\hline Left & Right & Mean & SD & Mean & SD & Mean & SD & Mean & SD \\
\hline \multicolumn{10}{|c|}{ Bird 16} \\
\hline 0 & 0 & 11.7 & 2.3 & 10.2 & 2.0 & 11.7 & 1.9 & 10.2 & 1.9 \\
\hline 12 & 12 & 13.4 & 2.7 & 13.1 & 3.2 & 14.7 & 1.9 & 15.4 & 3.2 \\
\hline 15 & 15 & 15.8 & 4.0 & 16.2 & 4.0 & 18.3 & 2.4 & 18.8 & 3.5 \\
\hline 18 & 15 & 17.3 & 3.5 & 16.3 & 4.0 & 20.9 & 2.2 & 19.0 & 4.6 \\
\hline 21 & 15 & 21.2 & 5.0 & 16.9 & 5.4 & 25.7 & 4.6 & 19.3 & 4.6 \\
\hline 18 & 18 & 19.0 & 3.3 & 18.2 & 5.3 & 21.7 & 1.9 & 22.8 & 5.4 \\
\hline 15 & 30 & 17.6 & 5.5 & 24.7 & 5.7 & 19.3 & 2.9 & 34.2 & 2.9 \\
\hline 30 & 45 & 31.8 & 8.2 & 37.6 & 11.2 & 36.8 & 6.1 & 50.1 & 4.6 \\
\hline 45 & 60 & 42.2 & 12.4 & 49.3 & 17.0 & 55.2 & 7.9 & 70.6 & 7.2 \\
\hline 15 & 60 & 29.2 & 14.6 & 44.2 & 12.1 & 24.5 & 10.1 & 66.3 & 5.3 \\
\hline 21 & 63 & 27.2 & 12.6 & 48.8 & 12.0 & 30.4 & 9.9 & 72.0 & 5.6 \\
\hline \multicolumn{10}{|c|}{ Bird 110} \\
\hline 0 & 0 & 13.2 & 3.1 & 13.1 & 2.7 & 12.9 & 3.0 & 12.7 & 2.6 \\
\hline 12 & 12 & 13.7 & 4.2 & 14.8 & 5.6 & 15.8 & 3.9 & 16.8 & 5.3 \\
\hline 15 & 15 & 16.8 & 4.9 & 16.6 & 4.0 & 18.2 & 2.8 & 19.2 & 2.7 \\
\hline 18 & 15 & 19.9 & 4.8 & 18.9 & 4.1 & 22.7 & 3.3 & 20.4 & 3.5 \\
\hline 21 & 15 & 24.3 & 7.0 & 19.8 & 4.1 & 27.8 & 5.0 & 20.0 & 3.0 \\
\hline 18 & 18 & 20.4 & 4.6 & 19.2 & 3.6 & 22.9 & 3.1 & 21.8 & 2.1 \\
\hline 15 & 30 & 27.6 & 7.1 & 28.6 & 7.0 & 27.3 & 6.5 & 35.3 & 5.0 \\
\hline 30 & 45 & 29.7 & 8.5 & 37.3 & 11.9 & 36.7 & 4.4 & 55.3 & 10.2 \\
\hline 15 & 30 & 20.7 & 4.3 & 28.8 & 5.8 & 20.9 & 4.0 & 34.9 & 4.0 \\
\hline 15 & 60 & 26.8 & 13.1 & 41.4 & 11.6 & 23.0 & 5.9 & 69.1 & 7.0 \\
\hline 63 & 21 & 41.9 & 13.1 & 32.2 & 17.3 & 68.9 & 5.4 & 32.8 & 15.2 \\
\hline \multicolumn{10}{|c|}{ Bird 143} \\
\hline 0 & 0 & 16.3 & 3.0 & 14.7 & 2.6 & 15.3 & 3.0 & 14.2 & 2.7 \\
\hline 12 & 12 & 15.9 & 3.5 & 15.9 & 3.4 & 16.1 & 2.7 & 16.4 & 3.0 \\
\hline 15 & 15 & 16.9 & 3.5 & 16.8 & 3.0 & 19.0 & 2.8 & 18.4 & 2.4 \\
\hline 15 & 18 & 17.3 & 3.2 & 17.4 & 3.1 & 18.8 & 2.4 & 20.9 & 2.2 \\
\hline 15 & 21 & 17.7 & 3.4 & 20.9 & 6.1 & 18.8 & 2.1 & 25.6 & 3.3 \\
\hline 18 & 18 & 17.1 & 2.8 & 18.0 & 3.3 & 20.4 & 2.0 & 21.4 & 2.4 \\
\hline 30 & 15 & 23.1 & 6.7 & 19.0 & 5.3 & 34.4 & 3.7 & 19.4 & 3.7 \\
\hline 45 & 30 & 30.4 & 8.9 & 27.6 & 11.5 & 51.4 & 4.3 & 39.6 & 5.0 \\
\hline 45 & 15 & 30.3 & 7.9 & 20.6 & 7.4 & 49.8 & 3.7 & 19.6 & 3.9 \\
\hline 60 & 15 & 33.8 & 12.3 & 23.2 & 11.1 & 65.6 & 5.6 & 19.1 & 4.1 \\
\hline 63 & 21 & 40.5 & 13.3 & 25.7 & 14.3 & 69.9 & 5.4 & 28.1 & 5.7 \\
\hline \multicolumn{10}{|c|}{ Bird 159} \\
\hline 0 & 0 & 10.9 & 2.4 & 12.4 & 2.8 & 11.0 & 2.9 & 11.8 & 1.9 \\
\hline 12 & 12 & 12.3 & 3.5 & 13.3 & 3.3 & 14.1 & 2.9 & 15.8 & 2.7 \\
\hline 15 & 15 & 13.8 & 3.3 & 12.6 & 2.8 & 18.0 & 2.6 & 17.1 & 1.7 \\
\hline 15 & 18 & 15.9 & 3.8 & 17.0 & 5.3 & 18.4 & 4.0 & 21.8 & 4.0 \\
\hline 15 & 21 & 16.2 & 4.9 & 18.1 & 4.9 & 19.2 & 5.4 & 24.7 & 4.3 \\
\hline 18 & 18 & 17.0 & 4.0 & 16.3 & 3.6 & 21.8 & 4.2 & 21.7 & 3.7 \\
\hline 30 & 15 & 21.8 & 6.7 & 18.4 & 5.7 & 34.6 & 3.4 & 20.6 & 5.3 \\
\hline 45 & 30 & 29.2 & 9.5 & 26.2 & 8.9 & 50.2 & 4.0 & 34.8 & 4.1 \\
\hline 45 & 15 & 27.7 & 9.1 & 21.2 & 9.1 & 48.7 & 2.5 & 21.3 & 6.4 \\
\hline 60 & 15 & 30.0 & 12.2 & 24.0 & 12.9 & 63.3 & 2.5 & 18.2 & 3.1 \\
\hline 21 & 63 & 23.8 & 10.8 & 33.3 & 13.1 & 29.6 & 5.8 & 69.0 & 2.8 \\
\hline
\end{tabular}

Note-All entries are given in seconds.

creased. The power functions fit the upper-bound data well and provided the closest fit of all the measures to the no-upper-bound results.

In summary, all of the quantitative analyses fared approximately equally well in describing the results of the upper-bound conditions. All did less well with the no-upper-bound conditions, but the best account was provided by reinforced duration.

\section{Interactions}

The same ratio durations were not recovered with successive exposure to a time requirement in the absence of upper bounds. The left column of Figure 5 shows that the ratio times produced by a given requirement were influenced by the time requirement prevailing in the other component. The ratio time always moved in the direction of the accom- 
Table 4

Upper Bound: Results

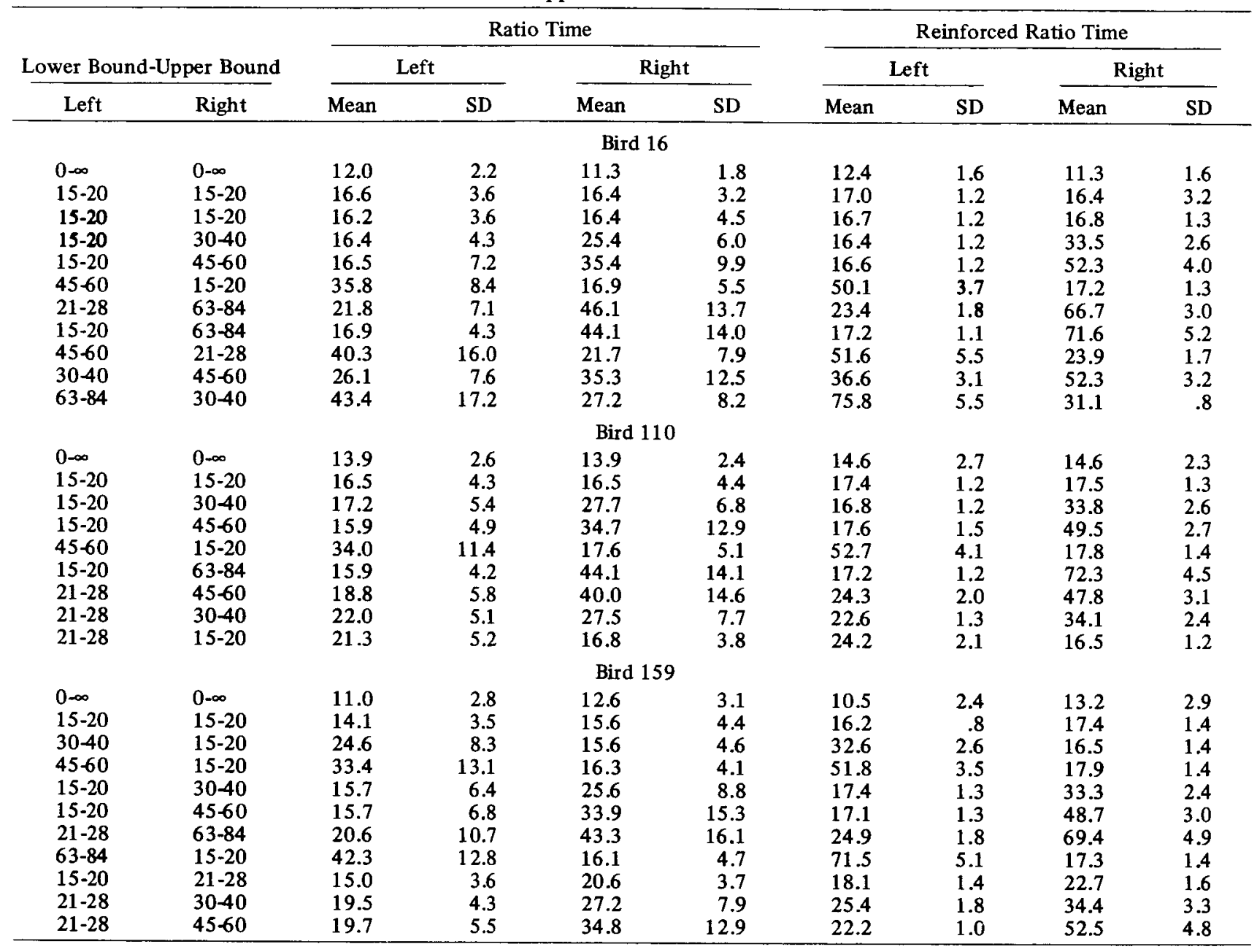

Note-Entries are given in seconds.

panying requirement. The larger the difference between requirements, the more the ratio times shifted. The right column of Figure 5 shows that with upper bounds induction between components was small at best, and in several cases, a given requirement produced recoverable performance. Reinforced ratio times generally showed the same sorts of interactions as did the overall ratio times (Tables 3 and 4).

Induction would have occurred if the pigeons sometimes responded in each component as if the requirement prevailed and sometimes as if the other component was in effect. The best support for such an interpretation would have been bimodal frequency distributions of ratio times with peaks occurring at the points characteristic of each of the two requirements. However, bimodality never was evident. In all cases, frequency distributions were unimodal or flat over a wide range. Interactions appeared to reflect a functional combination of the requirements rather than inconsistent control by each of the two.

The interactions were quantified by assuming that both forms of the temporal differentiation equation described the data accurately. Solving the equation for $t$ (or $R$ ) showed the values required to account for the results. These predicted values were assumed to represent the weighted average of the actual values according to the equations $t_{p}=w t_{r}+$ $(1-w) t_{o}$ and $r_{p}=w R_{T}+(1-w) R_{o}$, where $t_{p}$ and $R_{p}$ represent the predicted time requirement and reinforced duration, respectively, $t_{T}$ and $R_{T}$ are the requirement and the reinforced duration for the component under consideration, $t_{o}$ and $R_{o}$ are the time requirement and the reinforced duration for the other component, and $w$ is the weighting factor. Solving for $w$ allowed calculation of the weights needed to describe the data. 

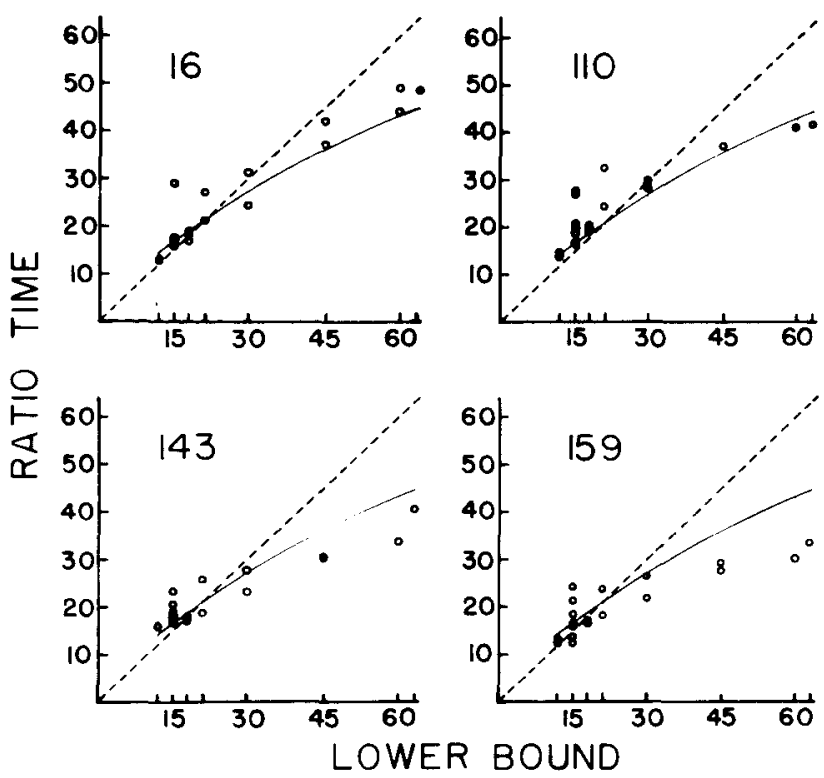

Figure 1. Mean ratio time for each duration requirement in the no-upper-bound conditions. The dashed lines indicate matching of ratio time to the requirement. The curved lines are the predictions from the temporal differentiation equation.
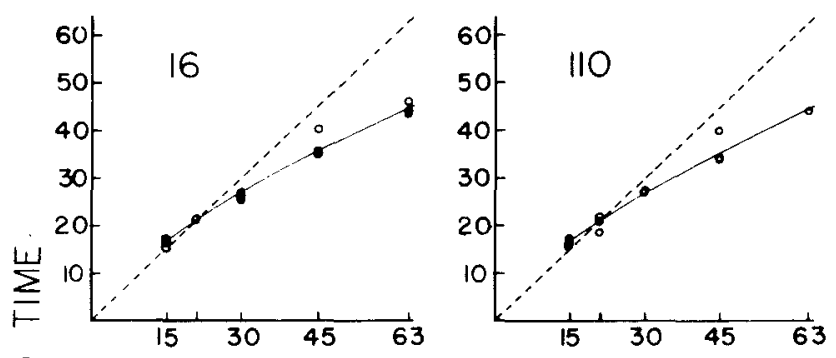

$\stackrel{0}{1}$

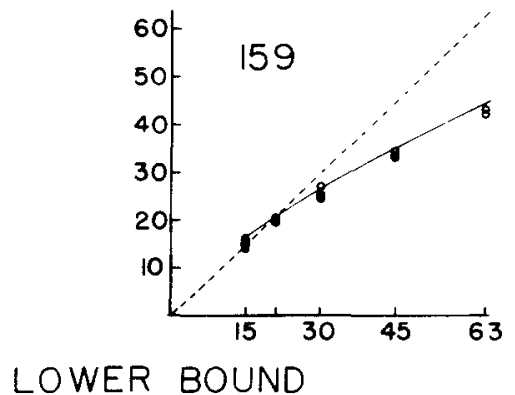

Figure 2. Mean ratio time for each duration requirement in the upper-bound conditions. Details as in Figure 1.

The weight was determined for each component for each pair of time requirements. A weight of 1.0 meant no interaction, and a weight of 0 meant total determination by the other component (total lack of independence). The value of $w$ could exceed 1.0 or be less than 0 if the predicted time requirements did not fall somewhere between the two component values, and the weight equation could not be solved at all if the two component values were the same. Only weights between 0 and 1.0 were used to determine the average weight. For the upper-bound conditions, the average weights for each bird were between .93 and .97 for the lower-bound version and between .87 and .97 for the reinforced duration version. For the no-upper-bound conditions, the average weights were between .54 and .72 for the lower-bound version and between .55 and .71 for the unreinforced duration version. The overall mean weights across birds for the upper-bound and noupper-bound conditions, respectively, were .95 and .63 (lower-bound version) and .93 and .62 (reinforced duration version). These data quantitatively confirmed Figure 5 in showing interactions that were weaker with upper bounds than without. They indicate that the prevailing component always received the most weight, and that relative weighting depended on the reinforcement variable of presence or absence of upper bounds. The functional time requirement is determined by differential weighting of the two separate time values rather than by either operating totally independently.

\section{Weber's Law}

Attempts to apply Weber's law to response timing stem from previously reported constancies in the coefficient of variation (standard deviation divided by the mean) over a range of requirements (Gibbon, 1977). The log-log plots of Figure 6 show the standard deviations as functions of the mean ratio times, and Table 5 indicates the parameters of the bestfitting power functions relating the means and standard deviations to the time requirement (see section labeled "Lower Bound Functions" for the parameters for each measure for each bird). Because the slopes of the functions for means were less than those for standard deviations, the slopes of the functions shown in Figure 6 had exponents greater than 1.0 , which means that the coefficient of variation increased as the means increased. The average coefficients (about .3) were in the same range as those observed in other experiments, but the average did not describe the individual points. Increasing coefficients also were manifested upon reanalysis of other individual subject data (DeCasper \& Zeiler, 1974, Experiment 2; Platt, 1979, Figure 1.1). The constant coefficients reported when the data from individual subjects were grouped seems to result from individual differences in slope and points of maximum curvature for the individuals. Since increasing coefficients with all but very small means have also been found with humans (Getty, 1975) and rats (Cantor \& Wilson, 1981), and even with means less than $.05 \mathrm{sec}$ with pigeons (Zeiler, Davis, $\&$ DeCasper, 1980), a variety of timing data do not conform to Weber's law. A higher degree of constancy can be obtained by assuming a fixed latency to begin timing (Church, Getty, \& Lerner, 1976) 
Table 5

Best-Fitting Functions

\begin{tabular}{|c|c|c|c|c|c|c|c|c|c|}
\hline \multirow[b]{2}{*}{ Bird } & & \multicolumn{4}{|c|}{ Lower Bound Functions } & \multicolumn{4}{|c|}{ Reinforced Duration Functions } \\
\hline & & $\mathbf{n}$ & $\begin{array}{c}\mathrm{T}=\mathrm{kt}^{\mathrm{n}} \\
\mathrm{S}_{\mathrm{T}}=\mathrm{kt}^{\mathrm{n}} \\
\mathrm{k}\end{array}$ & PVAF & $\begin{array}{c}\text { TDE } \\
\text { (Lower Bound) } \\
\text { PVAF }\end{array}$ & $\mathrm{n}$ & $\begin{array}{c}\mathrm{T}=\mathbf{k R}^{\mathbf{n}} \\
\mathbf{S}_{\mathrm{T}}=\mathbf{k S}_{\mathbf{k}} \mathbf{R}^{\mathrm{n}}\end{array}$ & PVAF & $\begin{array}{c}\text { TDE } \\
\text { (Reinforced) } \\
\text { PVAF } \\
\end{array}$ \\
\hline \multicolumn{10}{|c|}{ No Upper Bound } \\
\hline 16 & $\begin{array}{l}\text { Mean } \\
\text { SD }\end{array}$ & $\begin{array}{l}.74 \\
.79\end{array}$ & $\begin{array}{l}2.3 \\
.54\end{array}$ & $\begin{array}{l}91 \\
52\end{array}$ & 88 & $\begin{array}{l}.83 \\
.98\end{array}$ & $\begin{array}{l}1.5 \\
1.5\end{array}$ & $\begin{array}{l}96 \\
67\end{array}$ & 91 \\
\hline 143 & $\begin{array}{l}\text { Mean } \\
\text { SD }\end{array}$ & $\begin{array}{l}.49 \\
.76\end{array}$ & $\begin{array}{c}4.7 \\
.56\end{array}$ & $\begin{array}{l}89 \\
41\end{array}$ & 71 & $\begin{array}{r}.56 \\
1.65\end{array}$ & $\begin{array}{r}3.5 \\
.8\end{array}$ & $\begin{array}{l}92 \\
94\end{array}$ & 85 \\
\hline 159 & $\begin{array}{l}\text { Mean } \\
\text { SD }\end{array}$ & $\begin{array}{l}.50 \\
.68\end{array}$ & $\begin{array}{c}4.2 \\
.75\end{array}$ & $\begin{array}{l}79 \\
45\end{array}$ & 26 & $\begin{array}{l}.58 \\
.32\end{array}$ & $\begin{array}{l}2.9 \\
4.1\end{array}$ & $\begin{array}{r}83 \\
0\end{array}$ & 56 \\
\hline \multicolumn{10}{|c|}{ Upper Bound } \\
\hline 159 & $\begin{array}{l}\text { Mean } \\
\text { SD }\end{array}$ & $\begin{array}{l}.71 \\
.88\end{array}$ & $\begin{array}{l}2.3 \\
.41\end{array}$ & $\begin{array}{l}99 \\
80\end{array}$ & 99 & $\begin{array}{l}.72 \\
.80\end{array}$ & $\begin{array}{l}2.0 \\
4.0\end{array}$ & $\begin{array}{l}99 \\
72\end{array}$ & 99 \\
\hline
\end{tabular}

Note- $T=$ mean ratio time; $S_{T}=$ standard deviation of all ratio times; $R=$ mean reinforced ratio time; $S_{R}=$ standard deviation of reinforced ratio times; $t=$ time requirement; $k, n=$ fitting parameters; $P V A F=$ percent variance accounted for; TDE $=$ temporal difference equation.

and adjusting each coefficient accordingly. This approach, however, has been questioned on both empirical and conceptual grounds (Cantor \& Wilson, 1981). The increasing coefficients observed prior to application of any adjusting strategy are predicted by an information theory analysis of timing (Cantor \& Wilson, 1981) as well as by a model that considers the results to be the outcome of timing and nontiming processes (Zeiler, 1981) rather than as the product of Weber's law.

In his argument for scalar timing, Gibbon (1977) concluded that the exponent of the power function relating the means of all response durations to those of reinforced durations should be similar to that describing the relation between the standard deviations. Table 5 (section labeled "Reinforced Duration
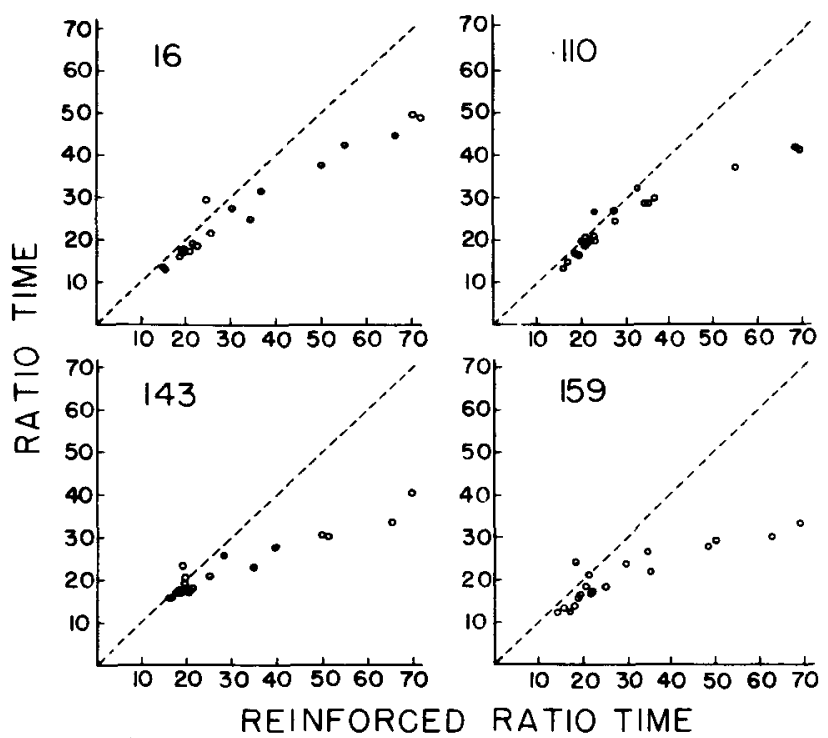

Figure 3. Mean ratio time as a function of mean reinforced ratio time in the no-upper-bound conditions. The dashed lines indicate matching of all ratio times to reinforced ratio times.
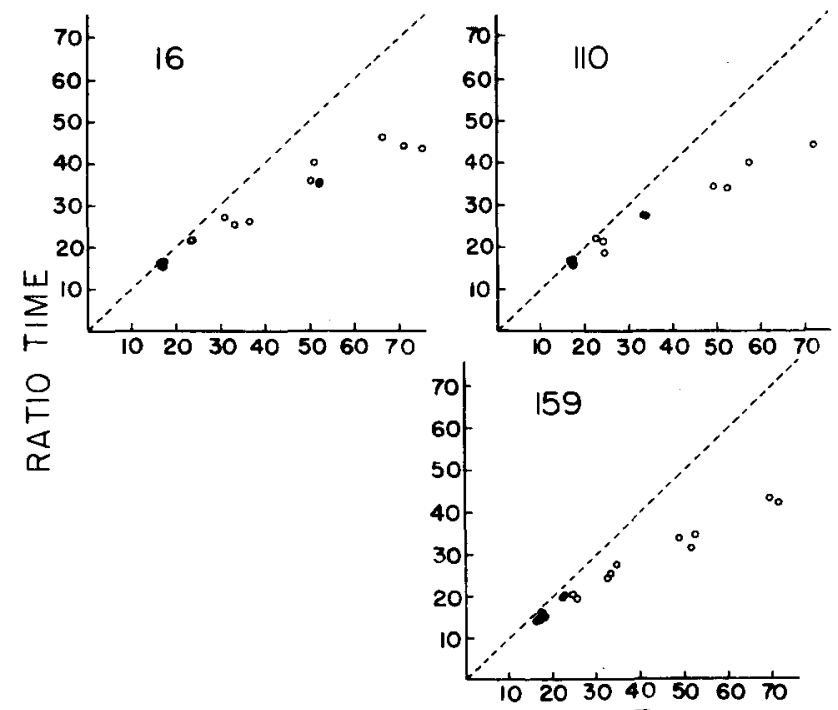

REINFORCED RATIO TIME

Figure 4. Mean ratio time as a function of mean reinforced ratio time in the upper-bound conditions. Details as in Figure 3. 


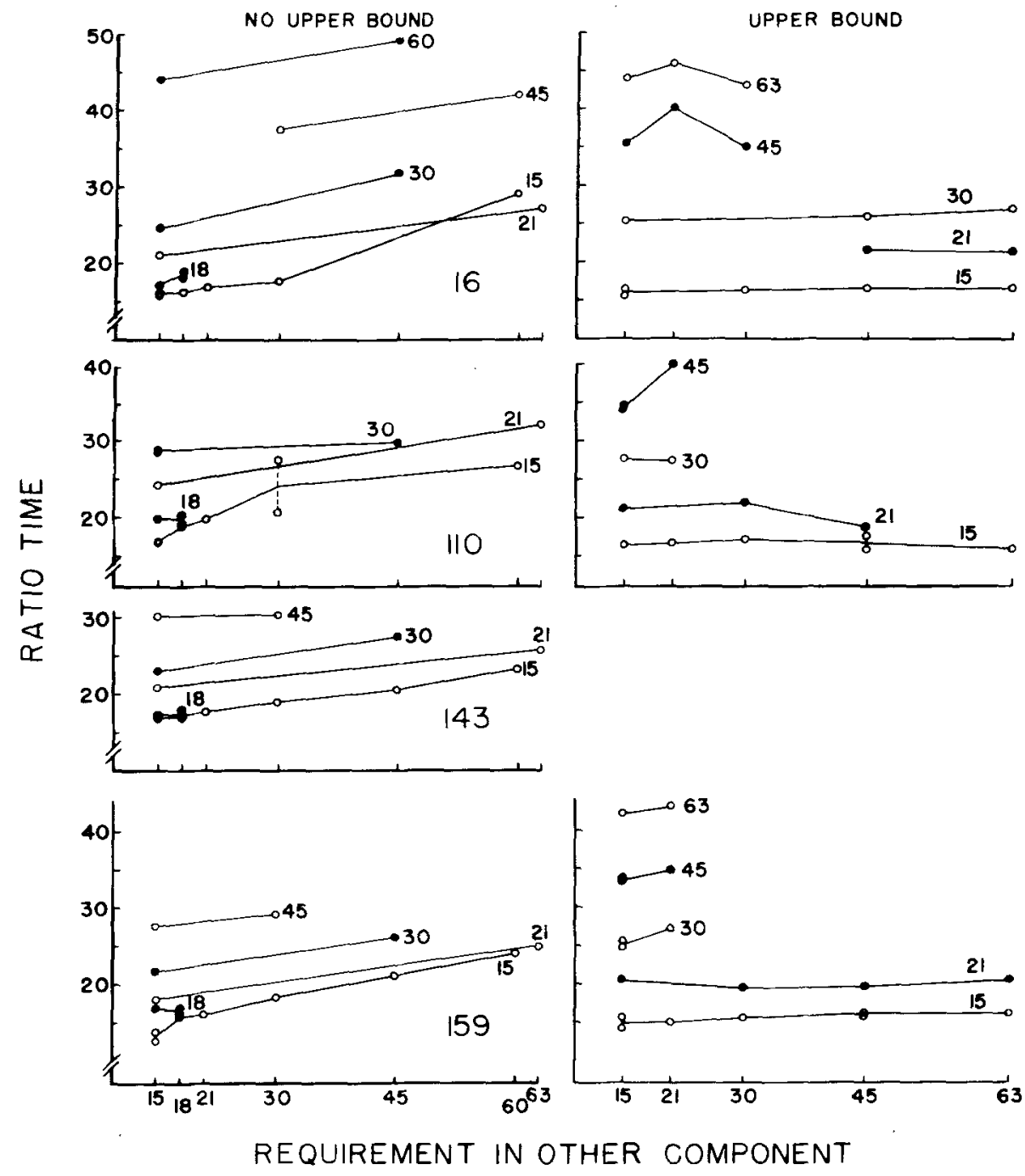

Figure 5. Mean ratio time in one component as a function of the duration requirement in the other component. The numerals adjacent to each curve show the duration requirement.

Functions") shows that the two exponents for individual pigeons differed by at least .08 and by as much as 1.09. Across pigeons and conditions, the average exponent for the means was .69 and that for the standard deviations was .88. Therefore, neither individual nor grouped data supported this version of the scalar timing hypothesis.

\section{DISCUSSION}

The context in which a time requirement appeared (the presence of another requirement; the presence or absence of an upper bound) exerted an impact on response timing. The effects were manifested as a weighted compromise of the current duration requirement with that prevailing in the other component, with the magnitude of the compromise determined by the presence or absence of the upper bound. The present description of contextual determination in temporal differentiation can be seen as another example of integration processes manifested in other areas of psychology (Anderson, 1981; Helson, 1964). That a different algebraic account of integration might do as well as or better than the presently employed weighted-averaging model is not of present concern, although it should be in the future; the point is that integration of separate stimuli operates generally, and the present results fit a commonly applicable quantitative description.

The fact that upper bounds diminished the magnitude of the interaction corresponds to numerous demonstrations that other variables influence the degree of independence of events (see Anderson, 1981; Helson, 1964). The present data suggest that weighted integration of these events may occur whenever the behavior appropriate to the longer require- 


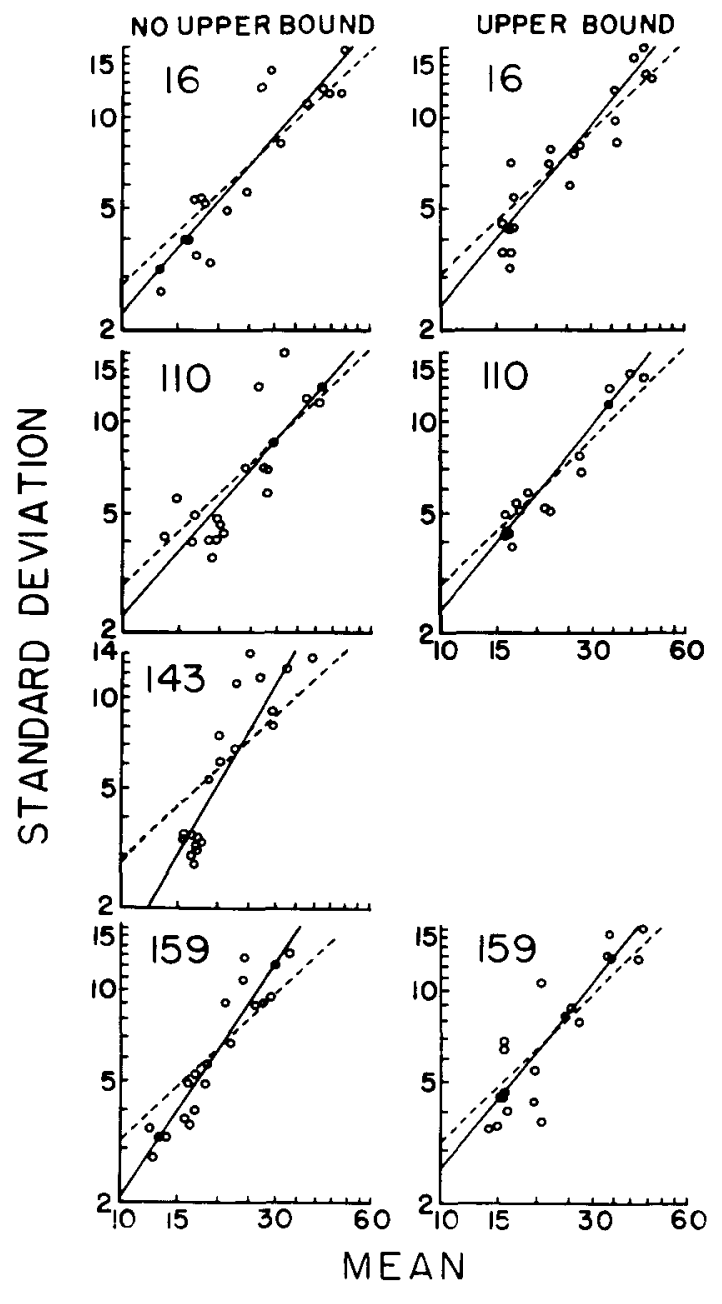

Figure 6. Standard deviation of all ratio times as a function of mean ratio time. The dashed lines show the predicted points for a constant coefficient of variation, with the constant taken as the mean coefficient. The solid lines show the best-fitting power functions.

ment of a pair also is appropriate to the shorter requirement. Symmetry apparently is not essential, because the behavior generated by the shorter requirement is not likely to fit the longer. The noupper-bound conditions permitted this integration. The upper-bound conditions did so less readily, because the behavior appropriate to the long requirement often fell above the upper limit of the short. Integration, then, is subject to reinforcement variables, and is not a purely sensory phenomenon.

Functional integration raises the possibility that base duration (d) is itself part of the context determining the frame of reference. Base duration has been interpreted as a duration minimizing process (Platt, 1979), a persistent tendency to respond as if no time requirement prevails (Zeiler, 1981), and as the frame of reference for evaluating time requirements (DeCasper \& Zeiler, 1977). In the temporal differentiation equations, it appears once as a weighted factor and again as part of the exponent applied to the requirement or to the reinforced duration. Perhaps the double usage reflects two different roles, one serving as part of the stimulus integration process and the other as a duration-minimizing or response-persistence component.

Another question is whether timing aspects are the only factors entering into the integrated functional requirement. Data indicating that nontemporal features can influence timing (Thomas \& Cantor, 1978) suggest that the entire experimental situation may interact with temporal factors. The constants of the temporal differentiation equations may be incorporating such integration features, and their ability to describe so much data could reflect the high degree of similarity in experimental procedures. What part of the equations represents timing per se remains an open question.

\section{REFERENCES}

ANDERSON, N. H. Foundations of information integration theory. New York: Academic Press, 1981.

Cantor, M. B., \& Wilson, J. F. Temporal uncertainty as an associative metric: Operant simulations of Pavlovian conditioning. Journal of Experimental Psychology: General, 1981, 110, 232268.

Church, R. M., Getty, D. J., \& Lerner, N. D. Duration discrimination by rats. Journal of Experimental Psychology: Animal Behavior Processes, 1976, 2, 303-312.

DeCasper, A. J., \& Zeiler, M. D. Time limits for completing fixed ratios. III. Stimulus variables. Journal of the Experimental Analysis of Behavior, 1974, 22, 285-300.

DeCasper, A. J., \& Zeiler, M. D. Time limits for completing fixed ratios. IV. Components of the ratio. Journal of the Experimental Analysis of Behavior, 1977, 27, 235-244.

GETTY, D. J. Discrimination of short temporal intervals: A comparison of two models. Perception \& Psychophysics, 1975, 18, $1-8$.

GibBon, J. Scalar expectancy theory and Weber's law in animal timing. Psychological Review, 1977, 84, 279-325.

Helson, H. Adaptation-level theory. New York: Harper \& Row, 1964.

KuCH, D. O. Differentiation of press durations with upper and lower limits on reinforced values. Journal of the Experimental Analysis of Behavior, 1974, 22, 275-283.

MCDowell, J. J. Wilkinson's method of estimating the parameters of Herrnstein's hyperbola. Journal of the Experimental Analysis of Behavior, 1981, 35, 413-414.

PlatT, J. R. Temporal differentiation and the psychophysics of time. In M. D. Zeiler \& P. Harzem (Eds.), Reinforcement and the organization of behaviour. Chichester: Wiley, 1979.

Thomas, E. A. C., \& Cantor, N. E. Interdependence between the processing of temporal and non-temporal information. In $\mathbf{J}$. Requin (Ed.), Attention and performance VII. Hillsdale: Erlbaum, 1978.

Zeiler, M. D. Model of temporal differentiation. In C. M. Bradshaw, E. Szabadi, \& C. F. Lowe (Eds.), Quantification of steady-state operant behavior. Amsterdam: Elsevier/North Holland, 1981.

Zeiler, M. D., Davis, E. R., \& DeCasper, A. J. Psychophysics of key-peck duration in the pigeon. Journal of the Experimental Analysis of Behavior, 1980, 34, 23-33.

(Manuscript received September 7, 1982; revision accepted for publication January $21,1983$. ) 